\title{
Wild food plants and fungi used in the mycophilous Tibetan community of Zhagana (Tewo County, Gansu, China)
}

Jin Kang ${ }^{1,2 \dagger}$, Yongxiang Kang ${ }^{1 \dagger}$, Xiaolian $\mathrm{Ji}^{2}$, Quanping Guo ${ }^{3}$, Guillaume Jacques ${ }^{6}$, Marcin Pietras ${ }^{4,5}$, Nasim Łuczaj $^{7}$, Dengwu Li ${ }^{1}$ and Łukasz Łuczaj ${ }^{8^{*}+}$

\begin{abstract}
Background: The aim of the study was to investigate knowledge and use of wild food plants and fungi in a highland valley in the Gannan Tibetan Autonomous Region on the north-eastern edges of the Tibetan Plateau.

Methods: Field research was carried out in four neighbouring villages in a mountain valley of the Diebu (Tewo) county, surrounded by spruce forests. The study consisted of 30 interviews with single informants, or group interviews (altogether 63 informants). Apart from collecting voucher specimens, we also identified fungi using DNA barcoding.

Results: We recorded the use of 54 species of vascular plants. We also recorded the use of 22 mushroom taxa, which made up the largest category of wild foods. Fruits formed the largest category of food plants, with 21 species, larger than the wild greens category, which consisted of 20 species eaten after boiling or frying and 7 as raw snacks. We also recorded the alimentary use of 10 species of edible flowers and 3 species with underground edible organs. On average, 20.8 edible taxa were listed per interview (median - 21). The most listed category of wild foods was green vegetables (mean -7.5 species, median -8 species), but fruits and mushrooms were listed nearly as frequently (mean -6.3 , median -6 and mean $-5.8,-$ median 6 respectively). Other category lists were very short, e.g., flowers (mean - 1.3, median -1 ) and underground edible parts (mean - 0.7, median - 1). Wild vegetables are usually boiled and/or fried and served as side-dishes, or their green parts are eaten as snacks during mountain treks (e.g., peeled rhubarb shoots). Wild fruits are mainly collected by children and eaten raw, they are not stored for further use. The most widely used wild staple foods are Potetilla anserina roots, an important ceremonial food served on such occasions as New Year or at funerals. They are boiled and served with sugar and butter. The most important famine plants remembered by people are the aerial bulbils of Persicaria vivipara. Flowers are used as children's snacks - their nectar is sucked.
\end{abstract}

Conclusions: The number of wild taxa eaten in the studied valley is similar to that of other Tibetan areas. The structure of wild food plant taxa is also very typical for Tibetan speaking areas (e.g., the use of rhubarb shoots, Potentilla anserina, Persicaria vivipara). The studied community show a high level of mycophilia.

Keywords: Wild edible plants, Diebu (Tewo), Thebo, Ethnobotany, Ethnomycology, Edible mushrooms

\footnotetext{
* Correspondence: lukasz.luczaj@interia.pl

${ }^{\dagger}$ Equal contributors

${ }^{8}$ Department of Botany, Institute of Applied Biotechnology and Basic

Sciences, University of Rzeszów, Werynia 502, Kolbuszowa 36-100, Poland

Full list of author information is available at the end of the article
} 


\section{Background}

The Tibetan speaking population inhabits highland areas of Central Asia in five countries of the region, namely China, India, Bhutan, Nepal and Pakistan. Most Tibetans live in the People's Republic of China. Due to the inaccessibility of many Tibetan-inhabited areas and the political isolation of the region throughout the $20^{\text {th }}$ century, the number of ethnobotanical studies among Tibetans has been disproportionally small relative to the biocultural diversity present in the Tibetan Plateau and adjacent areas [1-7].

So far the wild food plants used by Tibetans have been documented in only a few studies. A team of scholars from Beijing recorded them in the Shangri-La region in Yunnan [2], Boesi researched Litang, Sichuan [1], and Kang and colleagues the Zhouqu county in Gansu [3]. We know even less about the edible fungi used by Tibetans, though existing publications show that in some communities at least a few taxa are widely collected and eaten $[1,3,4,8,9]$.

One of the least ethnobotanically explored parts of China is the province of Gansu, which lies in the northeastern part of the range of Tibetan languages and dialects. In previous expeditions some of the authors of this paper explored another area of western-central China, namely two valleys on the northern slope of the Qinling Mountains in Shaanxi [10,11], and one mountain valley in south-western Gansu inhabited by Tibetans [3]. Our previous study among the Tibetan people was performed in very unusual surroundings for this ethnic group, i.e., a relatively low elevation of around two thousand $\mathrm{m}$ a.s.l. In contrast, the study whose results we present in this article concerns a population of Gansu Tibetans living at higher altitudes (around $3000 \mathrm{~m}$ a.s.l.), among highland spruce forests.

The documentation of traditional wild foods is important for an understanding of traditional food systems [12-17] and for the cultural heritage of minorities living in China, as rapid economic progress increases food availability, and many lesser-used wild vegetables are becoming forgotten. Nowadays we still can interview many people who have good expertise on the emergency foods used during the 1959-61 famine (see e.g., [10, 11]). These people are 60-80 years old now, so in a few years there will be less of them and those remaining may be too old to go to the forest and show these foods. Eastern Asia is a particularly interesting region for the study of wild foods since nowhere in the world are such large numbers of species of wild vegetables used as in China, Thailand, Japan, Korea and neighbouring countries [17-24]. Tibetans living at high elevations, in areas with speciespoor floras, do not use as many species of wild vegetables, but their skill in utilizing local food resources is remarkable and worth documenting.
Tibetans are classified in China as one minority (Zangzu). However a mosaic of several Tibetan languages and dialects exists. In the study area Thebo (Diebu) Tibetan is spoken, which is not mutually intelligible with any of the neighbouring varieties, neither Amdo Tibetan, Chone (Zhuoni) nor Mbrugchu (Zhouqu). Few reliable sources exist on this dialect [25].

To fill the gap in the ethnobotanical exploration of the north-western part of China we aimed at documenting the use of wild food plants in one Tibetan valley in SW Gansu.

\section{Methods \\ Study site}

The province of Gansu in northwest China (Fig. 1.) has very diverse vegetation. It changes from desert in the north and centre through dry grasslands to deciduous forests in the mountainous south. In the south-west of Gansu, inhabited mainly by Tibetans, the Gannan Tibetan Autonomous Region was established.

Tibetans in Gannan constitute a very diverse collection of subsistence economies and speak a variety of dialects/ languages, still not sufficiently explored linguistically. In the northern part of the territory, in the grasslands, animal herders predominate. The Diebu (Tebo, Tewo) Tibetans, although living among forests, live both from animal husbandry and farming, whereas in the southern part of the region (Zhouqu), at lower elevations, plant cultivation is the main source of subsistence.

The studied valley is located along a small river valley (the river Yiwa), which is a tributary of Bailongjiiang (the White Dragon River). Bailongjiang valley and its surrounding areas constitute a mountainous forefront of the Tibetan Plateau. The dominant vegetation in the study area is forest, composed of Picea crassifolia Kom., Picea asperata Mast., Picea wilsonii Mast. Sabina tibetica Kom., Sabina saltuaria (Rehd. et Wils.) Cheng et W. T. Wang, Betula platyphylla Suk.etc. Other frequent types of vegetation are grassland and scrub (Beberis, Ribes, Rosa, Clematis and Rubus).

We studied Tibetan villages called Zhagana, near the source of Yiwa River, which is part of the township (zhen) of Yiwa, famous for its picturesque landscape (Figs. 1 and 2). These are: Daiba, Yeri, Dari and Dongwa (with a range of coordinates from: N 34 14 13", E $103^{\circ}$ $09^{\prime} 54^{\prime \prime}$ to N34 ${ }^{\circ} 14 \quad 14^{\prime \prime}$, E $103^{\circ} 10^{\prime} 58^{\prime \prime}$ ).

The Yiwa Township covers an area of $365 \mathrm{~km}^{2}$. The average annual rainfall is $447-762 \mathrm{~mm}$ (mainly in summer months), the annual average temperature is $5.7{ }^{\circ} \mathrm{C}$ (at altitude $2500 \mathrm{~m}$ a.s.l.) and $2.8^{\circ} \mathrm{C}$ (at altitude $3000 \mathrm{~m}$ ), and the frost-free period lasts on average 142 days per year [26]. The studied villages are purely Tibetan. The altitude at which the houses are located ranged from 2800 to $3300 \mathrm{~m}$ a.s.l. 

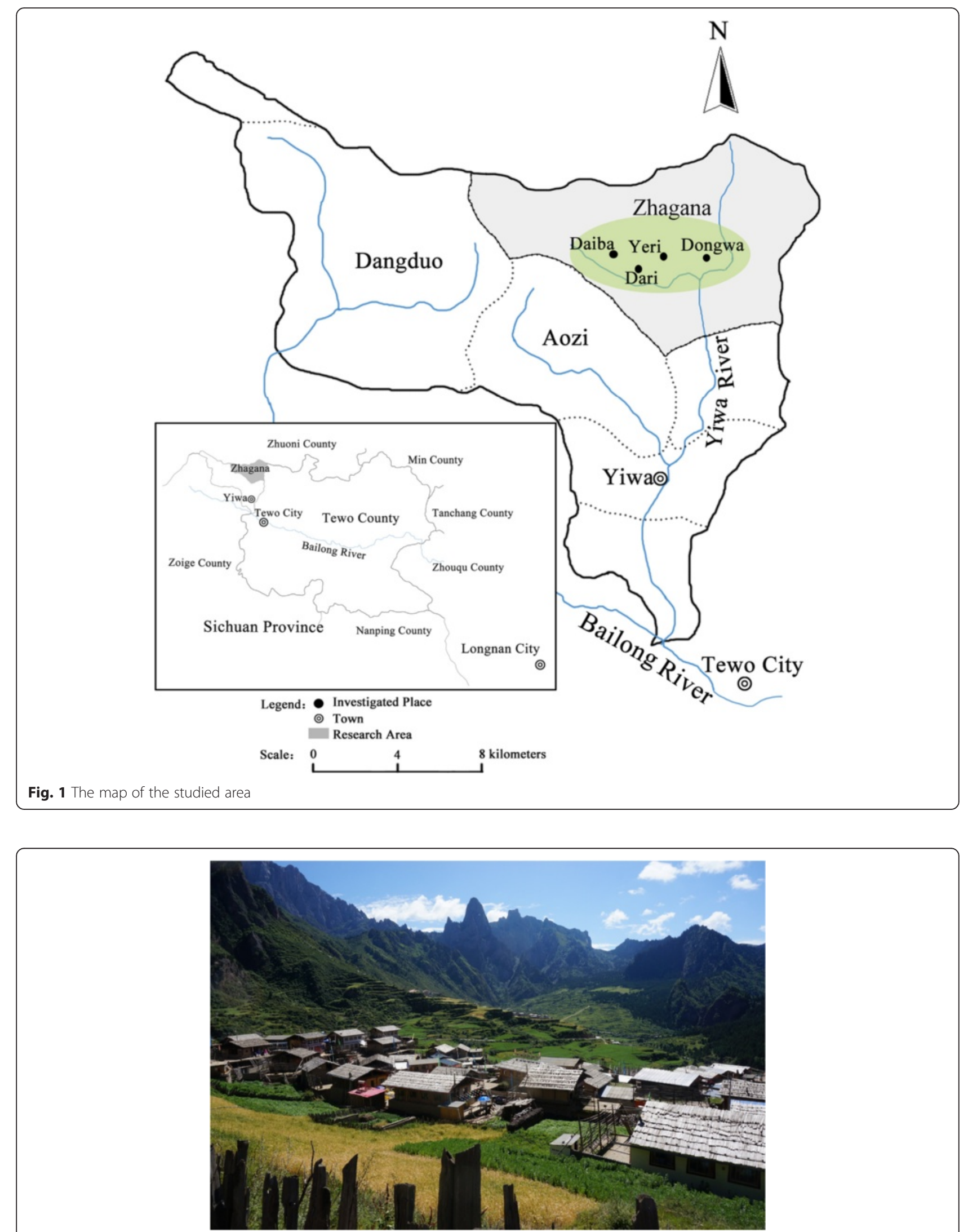

Fig. 2 A general view of Zhagana valley 
The studied population consists of subsistence farmers cultivating oats, barley and potatoes and keeping some yaks, cattle and pigs. Tourism has become an important part of the local economy as the valley has been voted one of the most picturesque places in China. This is causing a suddenly increasing influx of tourists (mainly from the capital of Gansu, Lanzhou). Over the last three years the local population have shifted their main activity from farming to building ad-hoc hiking shelters near many of the village homesteads. Apart from the pristine beautiful landscape, tourists are attracted to the fact that the village of Daiba was one of the headquarters of the famous American botanist explorer Joseph Rock who came to live there in 1925 or 1926 during his expedition to Tewo country (the house where he lived is marked and advertised as a tourist attraction - Fig. 3). Unfortunately Rock's materials from Gansu sunk with the ship which carried them from Calcutta to the USA during World War II [27-29].

\section{Data collection}

The field research was conducted in August 2015 using the Rapid Rural Appraisal approach [30, 31], and included 30 freelisting interviews (14 interviews - with 11 men and 3 women as single informants and 16 group interviews), which altogether involved 63 people (43 men and 22 women). The mean age of the participants was 55 (median 49, aged from 21 to 83). The research was carried out following the code of ethics of the American Anthropological Association [32] and the International Society of Ethnobiology Code of Ethics [33]. Oral prior informed consent was acquired.

The listed taxa (Table 1) were identified using specimens collected by informants in the forest or in the village. During freelisting we separately asked which species of wild vegetables (including underground organs), wild fruits and wild mushrooms were used. Making three separate freelists enabled comparison of the use of these categories and helped elicit answers from the respondents, who categorized the studied wild products in a similar way [34, 35]. Freelists were made orally and written down on the spot by our team. The conversations were additionally recorded using a digital sound recorder. The interviews were carried out in the local Thebo Tibetan dialect with the use of a local translator, or in Chinese if the interviewee could fluently speak this language. Many older men and nearly all middle-aged and elder women speak only Tibetan, whereas teenagers and young people in their twenties learned Mandarin Chinese at school and could facilitate communication. In contrast to their parents and grandparents they could also write down the plant names using Tibetan script. We recorded the local Tibetan names and transcribed them according to the International Phonetic Alphabet, and sound recordings of plant names were deposited in the Digital Repository of the University of Rzeszów [36].

Voucher specimens of fungi were deposited in the herbarium of the University of Warsaw (WA), and plants were deposited in the Herbarium of the Northwest A\&F University in Yangling (WUK). Plants were identified using the standard identification key concerning local floras, and their names follow the Plant List [37]. Fungi names follow the Index Fungorum [38].

Around half of the fungi specimens (those whose specimens were gathered) were successfully identified using the DNA barcoding technique [39, 40]. Fungal DNA was extracted from a small part of each fruiting body using a Plant and Fungi DNA Purification Kit (Eurx), following standard protocol. The PCR cocktail was composed of $4 \mathrm{ml}$ DNA extract, $0.5 \mathrm{ml}$ each of the primers (ITS5 and

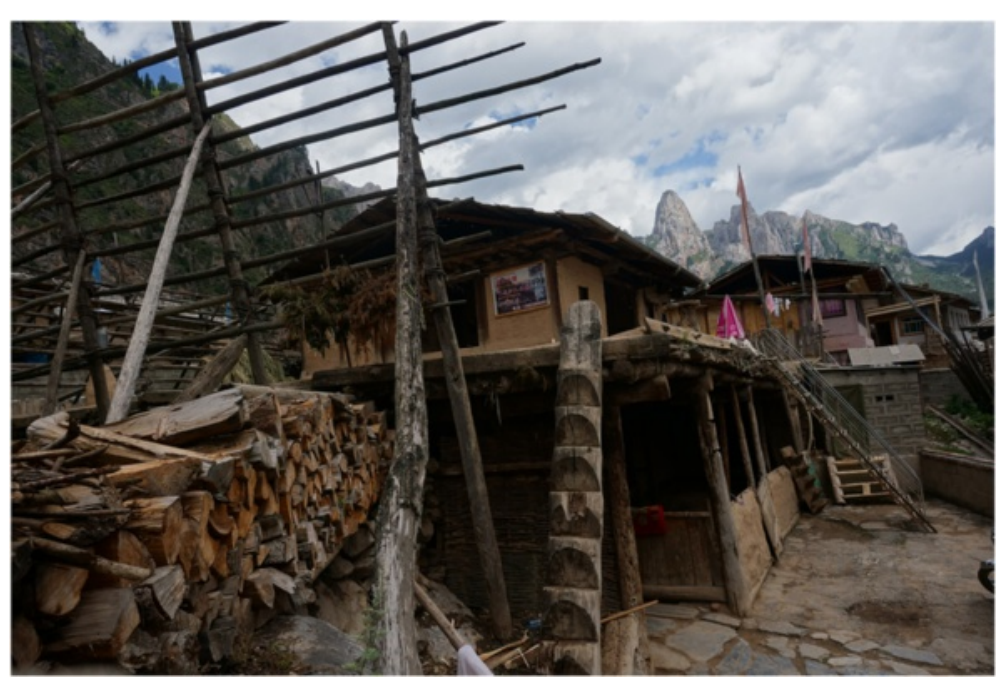

Fig. 3 The homestead where Joseph Rock once stayed 
Table 1 The list of fungi and plants used for food in Zhagana valley

\begin{tabular}{|c|c|c|c|c|c|}
\hline $\begin{array}{l}\text { Latin name checked in the } \\
\text { Plant List }\end{array}$ & $\begin{array}{l}\text { IPA pronunciation of local name } \\
\text { [etymology given in square brackets], } \\
\text { pronunciations without clear sound } \\
\text { recordings in () brackets }\end{array}$ & $\begin{array}{l}\text { Part } \\
\text { used }\end{array}$ & Preparation & $\begin{array}{l}\text { No. of } \\
\text { interviews } \\
\text { where } \\
\text { mentioned }\end{array}$ & $\begin{array}{l}\text { Specimen Number for fungi } \\
\text { it begins with WA } \\
00000517 \text {; for plants begins } \\
\text { with WUK Kang }\end{array}$ \\
\hline \multicolumn{6}{|l|}{ Fungi } \\
\hline $\begin{array}{l}\text { Agaricus campestris L. } \\
\text { (Agaricaceae) }\end{array}$ & po xá xa mbi & m & boiled / fried & 13 & 14 \\
\hline $\begin{array}{l}\text { Gomphus clavatus (Pers.) Gray } \\
\text { (Gomphaceae) }\end{array}$ & tढu tsá x̃̃ [square mushroom] & m & boiled / fried & 10 & 24 \\
\hline $\begin{array}{l}\text { Carathelasma imperiale (Quél.) } \\
\text { Singer (Tricholomataceae) }\end{array}$ & x̃̃ bo du & m & boiled / fried & 11 & 37 \\
\hline $\begin{array}{l}\text { Lactarius deliciosus (L.) Gray } \\
\text { var. deterrimus (Russulaceae) }\end{array}$ & X̃̃ má, X̃̃ má má [red mushroom] & m & $\begin{array}{l}\text { boiled / fried, dried for } \\
\text { winter, highly valued }\end{array}$ & 23 & 25,32 \\
\hline $\begin{array}{l}\text { Leucopaxillus giganteus } \\
\text { (Sowerby) Singer } \\
\text { (Tricholomataceae) }\end{array}$ & xõ kǎ: [white mushroom] & m & boiled / fried & 11 & 13,15 \\
\hline $\begin{array}{l}\text { Clitocybe fragrans (With.) P. } \\
\text { Kumm. (Tricholomataceae) } \\
\text { Russula aurea Pers., } \\
\text { Russula formula Jul. Schäff. } \\
\text { Russula sp. (at least } 3 \text { species } \\
\text { of Russula are used) } \\
\text { (Russulaceae) }\end{array}$ & si di Xõ [bird mushroom] & m & $\begin{array}{l}\text { boiled / fried, Russula } \\
\text { is eaten raw }\end{array}$ & 3 & $\begin{array}{l}\text { C. fragrans }-26, \text { R. formula } \\
-19,35, R \text {, aurea }-33, R . s p . \\
-27,38\end{array}$ \\
\hline an edible lichen & wo tsá lury & m & & 1 & - \\
\hline $\begin{array}{l}\text { Morchella elata Fr. } \\
\text { (Morchellaceae) }\end{array}$ & kú ku X̃̃ [cuckoo mushroom] & m & $\begin{array}{l}\text { boiled / fried, dried for } \\
\text { winter, mainly for sale, } \\
\text { highly valued }\end{array}$ & 11 & 10 \\
\hline $\begin{array}{l}\text { Auricularia auricula judae } \\
\text { (Bull.) Quél. (Auriculariaceae) }\end{array}$ & du: na $\mathbf{z u}(\mathrm{na}$ ju) $[\mathrm{du}=$ tree, naju = ear $]$ & m & $\begin{array}{l}\text { boiled / fried, dried for } \\
\text { winter }\end{array}$ & 10 & 17 \\
\hline Ramaria spp. (Gomphaceae) & $\begin{array}{l}\text { sa só si su [sasa are white forms, sisi - } \\
\text { yellow and mama - red ones, red is } \\
\text { best, white the worst] }\end{array}$ & m & $\begin{array}{l}\text { boiled / fried, highly } \\
\text { valued }\end{array}$ & 16 & $12,16,18,31,36$ \\
\hline $\begin{array}{l}\text { Sarcodon sp. (S. cf scabripes } \\
\text { (Peck) Banker?) (Bankeraceae) }\end{array}$ & lá xõ [eagle mushroom] & m & boiled / fried & 15 & 32 \\
\hline unidentified fugus & ta pu ta Xố & m & boiled / fried & 9 & - \\
\hline unidentified fugus & (po dzi h Xõ) & m & boiled / fried & 3 & - \\
\hline unidentified fugus & si Xõ & m & boiled / fried & 3 & - \\
\hline unidentified fugus & (Xõ dõ Zu) & m & boiled / fried & 10 & - \\
\hline unidentified fungus & 6a Xõ & $\mathrm{m}$ & boiled / fried & 2 & - \\
\hline unidentified fungus & ni mbi Xõ & m & boiled / fried & 3 & - \\
\hline unidentified fungus & t60 Xõ & m & boiled / fried & 1 & - \\
\hline unidentified fungus & dzaa si Xõ & $\mathrm{m}$ & boiled / fried & 4 & - \\
\hline unidentified fungus & (xõ tuo Zaoza) & m & boiled / fried & 1 & - \\
\hline \multicolumn{6}{|l|}{ Vascular plants } \\
\hline $\begin{array}{l}\text { Allium chrysanthum Regel } \\
\text { (Alliaceae) }\end{array}$ & ndzy ri, gu sI & $\mathrm{fl}, \mathrm{l}$ & $\begin{array}{l}\text { spice, dried for winter, } \\
\text { highly valued }\end{array}$ & 22 & 32 \\
\hline $\begin{array}{l}\text { Allium cyaneum Regel } \\
\text { (Alliaceae) }\end{array}$ & dză: & $\mathrm{fl}, \mathrm{l}$ & $\begin{array}{l}\text { spice, dried for winter, } \\
\text { highly valued }\end{array}$ & 18 & 37 \\
\hline Allium sp. (Alliaceae) & & $\mathrm{fl}, \mathrm{l}$ & spice & 1 & - \\
\hline $\begin{array}{l}\text { Allium sp. (white flowers) } \\
\text { (Alliaceae) }\end{array}$ & tça lá gu du & $\mathrm{fl}, \mathrm{l}$ & $\begin{array}{l}\text { spice, dried for winter, } \\
\text { highly valued }\end{array}$ & 15 & 45 \\
\hline Angelica of laxifoliata Diels & Đo tco lá & । & boiled / fried & 4 & 38 \\
\hline
\end{tabular}


Table 1 The list of fungi and plants used for food in Zhagana valley (Continued)

\begin{tabular}{|c|c|c|c|c|c|}
\hline Aralia chinensis L. (Araliaceae) & ka mó li ta & (buds) & boiled / fried & 1 & 26 \\
\hline Artemisia sp. (Compositae) & mo ta xa & । & boiled / fried & & - \\
\hline $\begin{array}{l}\text { Berberis circumserrata } \\
\text { (C.K.Schneid.) C.K.Schneid. } \\
\text { (Berberidaceae) }\end{array}$ & tsha ma si tshən, si tढ há & $\mid, f l$ & raw snack & 1 & 18 \\
\hline $\begin{array}{l}\text { Berberis potaninii Maxim. } \\
\text { (Berberidaceae) }\end{array}$ & tsha ma si tshon, si t6 há & $|, f|$ & raw snack & 1 & 21 \\
\hline Berberis sp. (Berberidaceae) & tsha ma si tshon, si t6 há & $|, f|$ & raw snack & 1 & 36 \\
\hline $\begin{array}{l}\text { Capsella bursa-pastoris (L.) } \\
\text { Medik. (Brassicaceae) }\end{array}$ & 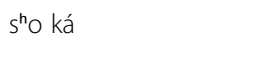 & L & boiled / fried & 1 & 40 \\
\hline $\begin{array}{l}\text { Carum buriaticum Turcz. } \\
\text { (Apiaceae) }\end{array}$ & dəm ${ }^{b}$ gu ni & $\mathrm{F}$ & $\begin{array}{l}\text { powdered, used as } \\
\text { sausage spice }\end{array}$ & 11 & 24 \\
\hline $\begin{array}{l}\text { Chenopodium album L. } \\
\text { (Amaranthaceea) }\end{array}$ & ni ló & L & boiled / fried & 15 & 10 \\
\hline Cirsium sp. (Asteraceae) & $\mathrm{t}^{\mathrm{h}} \mathrm{O}$ xá & L & boiled / fried & 2 & 39 \\
\hline $\begin{array}{l}\text { Elaeagnus rhamnoides (L.) } \\
\text { A.Nelson (Eleagnaceae) }\end{array}$ & la tá & $\mathrm{F}$ & raw snack & 10 & 11 \\
\hline $\begin{array}{l}\text { Epilobium angustifolium L. } \\
\text { (Onagraceae) }\end{array}$ & no t6o la & Sh & $\begin{array}{l}\text { boiled / fried, only } \\
\text { famine food }\end{array}$ & 1 & 33 \\
\hline Equisetum sp.? (Equisetaceae) & na dza ko rẽ & Sh & boiled / fried & 3 & - \\
\hline $\begin{array}{l}\text { Fragaria orientalis Losinsk. } \\
\text { (Rosaceae) }\end{array}$ & a ji sa & $\mathrm{F}$ & raw snack & 21 & 27 \\
\hline a Gentianaceae species & a nó ne, me tu pa pa & $\mathrm{N}$ & raw snack & 5 & - \\
\hline Lonicera sp. (Caprofoliaceae) & ra nó & $\mathrm{F}$ & raw snack & 12 & - \\
\hline $\begin{array}{l}\text { Ixeris chinensis (Thunb. ex } \\
\text { Thunb.) Nakai (Asteraceae) }\end{array}$ & $p^{\text {ha }}$ ki & L & boiled / fried & 7 & 30 \\
\hline $\begin{array}{l}\text { Mentha canadensis } \mathrm{L} \text {. } \\
\text { (Lamiaceae) }\end{array}$ & 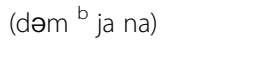 & L & boiled / fried & 1 & 25 \\
\hline $\begin{array}{l}\text { Notopterygium incisum } \\
\text { K.C.Ting ex H.T.Chang } \\
\text { (Apiaceae) }\end{array}$ & ฤó to & L & $\begin{array}{l}\text { boiled / fried, dried for } \\
\text { winter, highly valued }\end{array}$ & 23 & 35 \\
\hline Oxytropis sp. (Fabaceae) & rə nge ce li & $\mathrm{F}$ & famine food & 1 & 41 \\
\hline $\begin{array}{l}\text { Persicaria vivipara (L.) Ronse } \\
\text { Decr. (Polygonaceae) }\end{array}$ & ræ mbá & $\mathrm{F}$ & $\begin{array}{l}\text { seeds soaked } \\
\text { overnight to remove } \\
\text { bitterness }\end{array}$ & 20 & 12 \\
\hline $\begin{array}{l}\text { Picea asperata Mast. } \\
\text { (Pinaceae) }\end{array}$ & to tढ̆ & Ss & raw snack & & 44 \\
\hline Picea wilsonii Mast. (Pinaceae) & 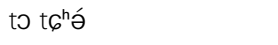 & Ss & raw snack & & 43 \\
\hline $\begin{array}{l}\text { Potentilla anserina } \mathrm{L} . \\
\text { (Rosaceae) }\end{array}$ & ts ̃̃ & $\mathrm{R}$ & $\begin{array}{l}\text { boiled with butter and } \\
\text { sugar/rice to make a } \\
\text { ceremonial dish, highly } \\
\text { valued }\end{array}$ & 17 & 16 \\
\hline $\begin{array}{l}\text { Prinsepia uniflora Batalin } \\
\text { (Rosaceae) }\end{array}$ & os $^{h_{i}}$ & $\mathrm{~F}$ & raw snack & 2 & 42 \\
\hline $\begin{array}{l}\text { Prunus salicifolia Kunth } \\
\text { (Rosaceae) }\end{array}$ & ţe lá & $\mathrm{F}$ & raw snack & 11 & 34 \\
\hline $\begin{array}{l}\text { Pteridium aquilinum (L.) Kuhn } \\
\text { var. latiusculum (Desv.) } \\
\text { Underw. ex A. Heller. } \\
\text { (Dennstaedtiaceae) }\end{array}$ & Gá la & L & $\begin{array}{l}\text { boiled / fried, dried for } \\
\text { winter, highly valued }\end{array}$ & 30 & 6 \\
\hline \multirow[t]{2}{*}{$\begin{array}{l}\text { Rheum officinale Baill. } \\
\text { (Polygonaceae) }\end{array}$} & lají & St & $\begin{array}{l}\text { raw snack on } \\
\text { mountain treks }\end{array}$ & 14 & 29 \\
\hline & tढ̆̂́, zo zế & St & & 13 & 19 \\
\hline
\end{tabular}


Table 1 The list of fungi and plants used for food in Zhagana valley (Continued)

\begin{tabular}{|c|c|c|c|c|c|}
\hline \multicolumn{3}{|l|}{$\begin{array}{l}\text { Rheum palmatum L. } \\
\text { (Polygonaceae) }\end{array}$} & \multicolumn{3}{|l|}{$\begin{array}{l}\text { raw snack on } \\
\text { mountain treks }\end{array}$} \\
\hline $\begin{array}{l}\text { Ribes alpestre Wall. ex Decne. } \\
\text { (Grossulariaceae) }\end{array}$ & $s^{\text {he }}$ ró & $F$ & raw snack & 17 & 9 \\
\hline $\begin{array}{l}\text { Ribes vilmorinii Jancz. } \\
\text { (Grossulariaceae) }\end{array}$ & shi nấ & $\mathrm{F}$ & raw snack & 13 & 7 \\
\hline $\begin{array}{l}\text { Rosa omeiensis Rolfe } \\
\text { (Rosaceae) }\end{array}$ & sa ka də la zó & Sh & raw snack & 8 & 28 \\
\hline $\begin{array}{l}\text { Rubus amabilis Focke } \\
\text { (Rosaceae) }\end{array}$ & $\mathrm{s}^{\mathrm{h} i}$ mã́ & $\mathrm{F}$ & raw snack & 18 & 8 \\
\hline $\begin{array}{l}\text { Rubus pileatus Focke } \\
\text { (Rosaceae) }\end{array}$ & $\mathrm{s}^{\mathrm{h} i}$ mã́ & $\mathrm{F}$ & raw snack & $x$ & 2 \\
\hline $\begin{array}{l}\text { Rubus pungens Cambess } \\
\text { (Rosaceae). }\end{array}$ & $\mathrm{s}^{\mathrm{h} i}$ mấ & $\mathrm{F}$ & raw snack & $x$ & 3 \\
\hline $\begin{array}{l}\text { Rubus xanthocarpus Bureau \& } \\
\text { Franch. (Rosaceae) }\end{array}$ & $s^{h} \tilde{I}$ & $\mathrm{~F}$ & raw snack & 15 & 4 \\
\hline $\begin{array}{l}\text { Rumex acetosa L. } \\
\text { (Polygonaceae) }\end{array}$ & a rá sa mbu & L & raw snack & 13 & 14 \\
\hline Salix spp. (Salicaceae) & tढấ lă & $\mathrm{FI}$ & $?$ & 3 & - \\
\hline $\begin{array}{l}\text { Salvia przewalskii Maxim. } \\
\text { (Lamiaceae) }\end{array}$ & $\begin{array}{l}\text { də mbə rá nə, a ne nə nə [goat } \\
\text { nipples] }\end{array}$ & $\mathrm{N}$ & raw snack & 12 & 17 \\
\hline $\begin{array}{l}\text { Sinopodophyllum hexandrum } \\
\text { (Royle) T.S.Ying } \\
\text { (Berberidaceae) }\end{array}$ & 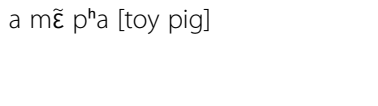 & $F$ & raw snack & 11 & 22 \\
\hline $\begin{array}{l}\text { Sonchus arvensis L. } \\
\text { (Asteraceae) }\end{array}$ & $p^{\text {ha }}$ ki [pig grass] & L & boiled / fried & 7 & 46 \\
\hline $\begin{array}{l}\text { Sorbus koehneana C.K.Schneid } \\
\text { (Rosaceae) }\end{array}$ & dzə mə ga ఢí & F & raw snack & 11 & 20 \\
\hline $\begin{array}{l}\text { Sorbus tianschanica Rupr. } \\
\text { (Rosaceae) }\end{array}$ & dzə mə ga бí & F & raw snack & 11 & 1 \\
\hline $\begin{array}{l}\text { Stachys affinis Bunge. } \\
\text { (Lamiaceae) }\end{array}$ & kª tə́ tso:wá & $\mathrm{R}$ & $\begin{array}{l}\text { tubers boiled in the } \\
\text { past }\end{array}$ & 2 & 15 \\
\hline $\begin{array}{l}\text { Stellera chamaejasme L. } \\
\text { (Lamiaceae) }\end{array}$ & ra rə tcá & R & $\begin{array}{l}\text { famine food in the } \\
\text { past }\end{array}$ & 1 & 31 \\
\hline $\begin{array}{l}\text { Thlaspi arvense L. } \\
\text { (Brassicaceae) }\end{array}$ & dəmbə tsă & $L$ & $\begin{array}{l}\text { seeds pressed for } \\
\text { temple oil }\end{array}$ & 14 & 5 \\
\hline $\begin{array}{l}\text { Triosteum pinnatifidum Maxim. } \\
\text { (Caprifoliaceae) }\end{array}$ & rə gu ji do & $\mathrm{F}$ & raw snack & 4 & 23 \\
\hline Urtica dioica L. (Urticaceae) & sá tsə & L & $\begin{array}{l}\text { boiled / fried, also for } \\
\text { dumpling filling and as } \\
\text { cure for swollen legs }\end{array}$ & 15 & 13 \\
\hline $\begin{array}{l}\text { unidentified plant from } \\
\text { grasslands }\end{array}$ & no rə ba & L & boiled / fried & 5 & - \\
\hline unidentified plant from & a ló ji lo & L & boiled / fried & 4 & - \\
\hline
\end{tabular}

$f$ fruits, $f$ flowers, $I$ leaves or buds, $m$ mushroom, $n$ nectar sucked, $r$ roots, rhizomes, tubers or bulbs, sh asparagus-like young shoots, ss solidified sap (resin), st peeled stalks

ITS4 in $10 \mathrm{nmol}$ concentration) and $5 \mathrm{ml}$ Type-it Microsatellite PCR Kit (Qiagen). PCR was performed using the following thermocycling conditions: an initial 15 min at $95{ }^{\circ} \mathrm{C}$, followed by 35 cycles at $95{ }^{\circ} \mathrm{C}$ for $30 \mathrm{~s}$, $55{ }^{\circ} \mathrm{C}$ for $30 \mathrm{~s}, 72{ }^{\circ} \mathrm{C}$ for $1 \mathrm{~min}$, and a final cycle of $10 \mathrm{~min}$ at $72{ }^{\circ} \mathrm{C}$. PCR products were estimated by running $5 \mathrm{ml}$ DNA amplicon on $1.5 \%$ agarose gel for $30 \mathrm{~min}$. The
PCR products were sequenced with the use of ITS4 primers at the Laboratory of Molecular Biology of Adam Mickiewicz University (Poznań). The obtained sequences were verified visually on chromatograms using BIOEDIT. Nuclear ITS sequences obtained in this study are deposited in GenBank [41], with the accession numbers listed in Table 2. 
Table 2 The results of DNA barcoding

\begin{tabular}{lllll}
\hline Molecular identification & Accession number & Best match sequence / accession number & E-value & Sequence Similarity (\%) \\
\hline Agaricus campestris & KX008974 & Agaricus campestris FJ223223 & 0.0 & 98 \\
Ramaria sp.2 & KX008975 & Ramaria sp. UDB024083 & 0.0 & 97 \\
Lactarius deliciosus var. deterrimus & KX008976 & Lactarius deliciosus EF685060 & 0.0 & 99 \\
Lactarius deliciosus var. deterrimus & KX008977 & Lactarius deliciosus EF685060 & 0.0 & 99 \\
Russula sp. & KX008978 & Russula sp. EU057100 & 0.0 & 98 \\
Auricularia auricula-judae & KX008979 & Auricularia auricula-judae FJ478123 & 0.0 & 100 \\
Morchella elata & KX008980 & Morchella elata GQ249054 & 0.0 & 100 \\
Sarcodon sp. & KX008981 & Sarcodon scabripes JN135191 & 0.0 & 96 \\
Leucopaxillus giganteus (Sowerby) Singer & KX008982 & Leucopaxillus giganteus JQ639151 & 0.0 & 98 \\
Leucopaxillus giganteus (Sowerby) Singer & KX008983 & Leucopaxillus giganteus JQ639151 & 0.0 & 99 \\
Ramaria sp. 3 & KX008984 & Ramaria cf coulterae JX310420 & 0.0 & 94 \\
Russula firmula & KX008985 & Russula firmula KJ867373 & 0.0 & 99 \\
Russula sp. & KX008986 & Russula sp. LC035243 & 0.0 & 99 \\
Catathelasma imperiale & KX008987 & Catathelasma imperiale UDB007898 & 0.0 & 97 \\
Gomphus clavatus & KX008988 & Gomphus clavatus UDB011658 & 0.0 & 99 \\
Ramaria sp.1 & KX008989 & Ramaria sp. UDB013130 & 0.0 & 94 \\
Ramaria sp.1 & KX008990 & Ramaria sp. UDB013130 & 0.0 & 94 \\
Ramaria sp1 & KX008991 & Ramaria sp. UDB013130 & 9.0
\end{tabular}

\section{Results}

Among the twelve most frequently mentioned wild foods are five species of wild vegetables - Pteridium aquilinum (L.) Kuhn (Fig. 4), Notopterygium incisum K.C.Ting ex H.T.Chang (Fig. 5), Allium chrysanthum Regel (Fig. 6), A. cyaneum Regel and Chenopodium album L., two taxa of fungi Lactarius deliciosus and Ramaria spp. (Fig. 7), three species of fleshy fruits (Fragaria orientalis Losinsk, Rubus spp., Ribes alpestre Wall. ex Decne) and two species used as staple foods (Persicaria vivipara (L.) Ronse Decr. syn. Polygonum viviparum L., edible seeds) and Potentilla anserina L. (edible tubers, Fig. 8).

We recorded the use of 54 species of vascular plants (Table 1). We also recorded the use of 22 mushroom taxa (Tables 1 and 2), which formed the largest category of wild foods. Fruits formed the largest category of food plants, with 21 species, larger than the wild greens category, which included 20 species eaten after boiling or

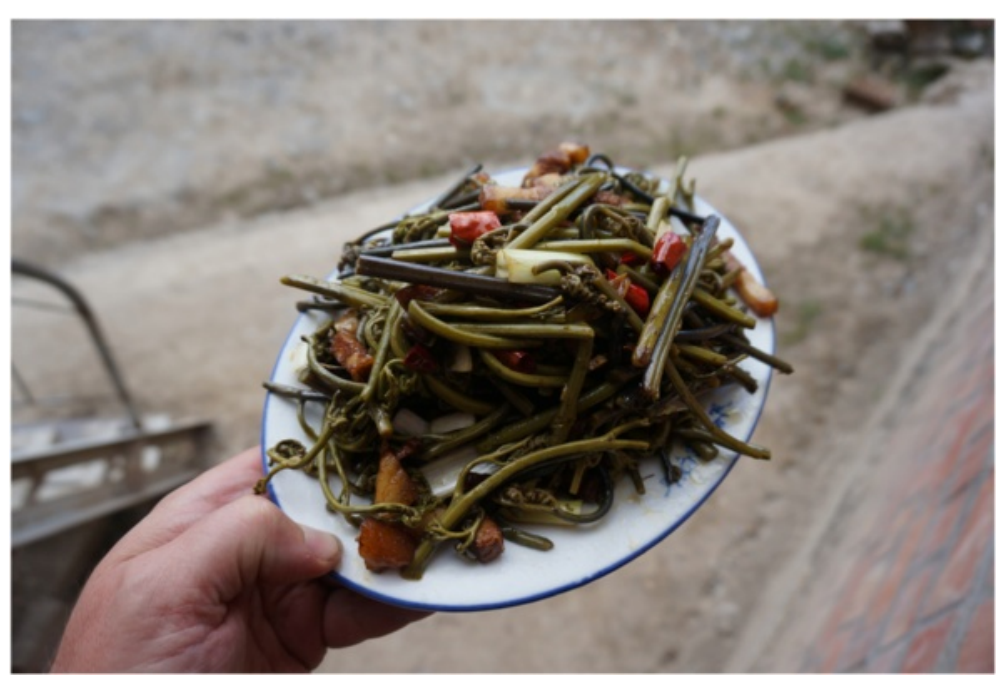

Fig. 4 Fried Pteridium fronds. The fronds were dried and reconstituted in water before frying 


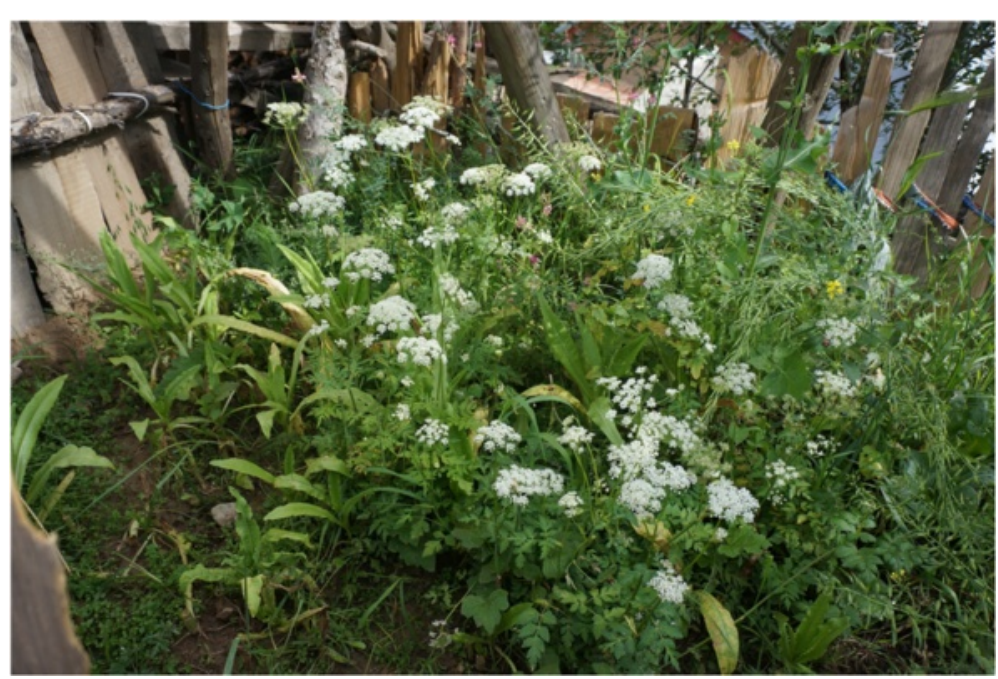

Fig. 5 Nothopterigium is sometimes planted in home gardens as it is appreciated for its celery-like fragrance

frying and 7 as raw snacks. We also recorded the alimentary use of 10 species of edible flowers and 3 species with underground edible organs. On average, 20.8 edible taxa were listed per interview (median - 21). The most-listed category of wild foods was green vegetables (mean -7.5 species, median -8 species), but fruits and mushrooms were listed nearly as frequently (mean -6.3 , median -6 and mean -5.8 , median -6 respectively). Other category lists were very short: flowers (mean -1.3 , median -1 ), underground edible parts (mean -0.7 , median -1 ).

Most wild vegetables and mushrooms are usually boiled, sprinkled with hot oil and served as side-dishes. Wild fleshy fruits are collected mainly by children and eaten raw. Some green parts of plants are eaten as raw snacks: plants with a sour taste (Rumex leaves, Rheum peeled stalks), solidified spruce sap and nectar sucked out of flowers.

In times of famine or grain scarcity Persicaria vivipara fruits were mixed with barley and used to make flour. This was practiced even up until the 1980s. Other wild staples are the small tubers of Potentilla anserina. They are still gathered now, but are treated only as ceremonial foods, being served during New Year celebrations, funerals and other ceremonial occasions. Their rarer use stems from a very tedious gathering procedure. The tubers are dug out by women in late autumn or early spring. One woman can gather $0.5-1 \mathrm{~kg}$ of tubers per day. In the past they also constituted emergency food. Several informants observed changes in the frequency with which wild foods are collected: adults collect and

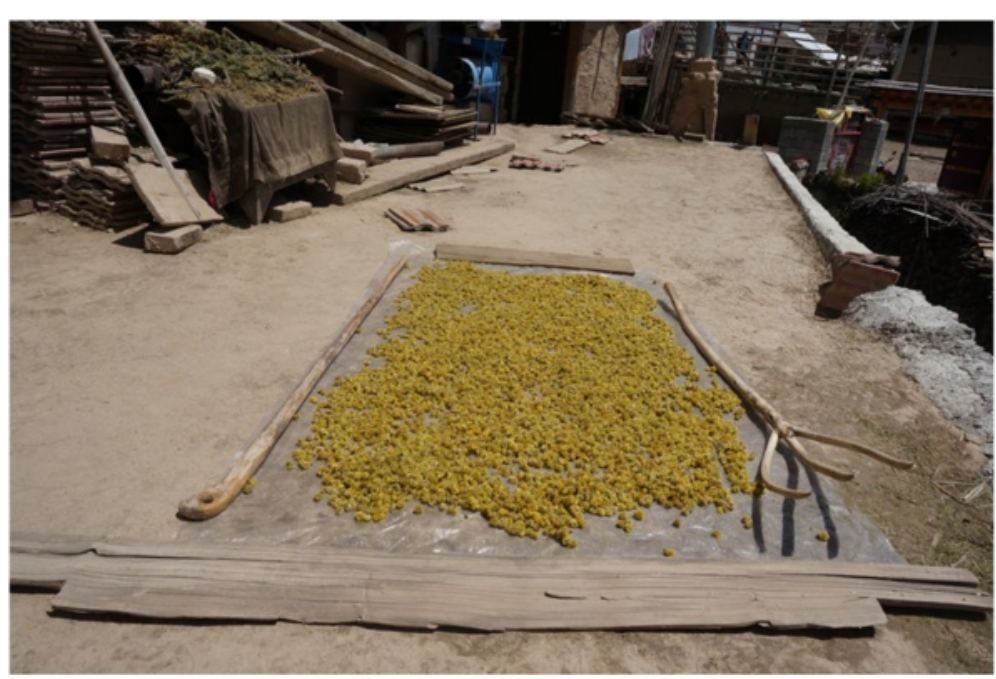

Fig. 6 Allium chrysanthum flowers dried in the sun for winter 


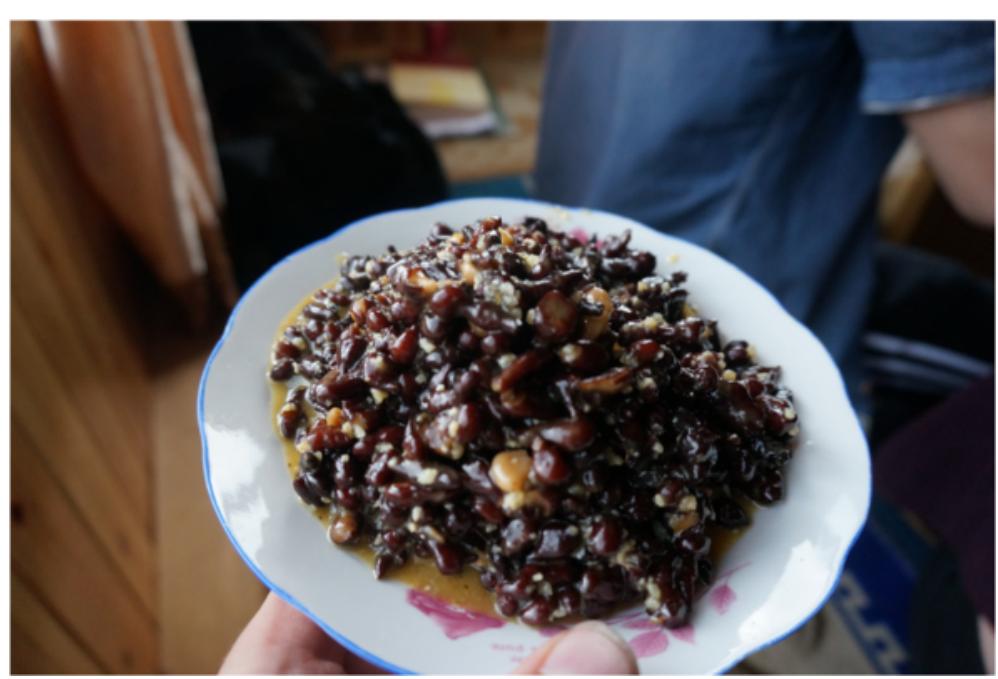

Fig. 7 Potentilla anserina tubers boiled and served with butter and sugar. This is an expensive traditional dish served on important holidays

eat less wild vegetables and children snack less on wild fruits. Most people usually use only a few wild vegetables, such as Allium spp., Pteridium and Notopterygium. Some people have stopped eating Chenopodium and Urtica. Due to the increasing involvement of tourism in the valley in the last 5 years, people do not have time to gather fungi in summer, at the peak of the tourist season.

Practically all families dry wild vegetables for later use, however they do not lacto-ferment them. People usually dry bracken (Pteridium) fronds, Nothopterigium leaves and wild garlic (Allium) flower heads. They also dry a few species of mushrooms, mainly morels (Morchella conica; Fig. 9) and milk velvet caps (Lactarius deliciosus var. deterrimus). Morels are an important article of commerce, as is the medicinal Cordyceps sinensis mushroom, which was regarded by our informants as medicinal and not an edible mushroom. Some of our informants stored a few large sacks of morels for sale.

\section{Discussion}

The few plant names available in the only other existing paper dealing with Thebo vocabulary [25] are very close to those recorded during the field trip, for instance Potentilla anserina $\left(\mathrm{tso}^{\mathrm{L}}\right.$ in $[25], \mathrm{tso}^{\mathrm{L}}$ in the recordings, from Old Tibetan gro.ma) and the word for mushroom ( $\mathrm{x}^{\mathrm{h}}$ awũ in [25], Xõ in the recordings, from Old Tibetan ca.mo). Note that the sound change from Old Tibetan gr- to Thebo tsin the word for Potentilla anserina is unique, among the varieties spoken in this region, to the Thebo language (Chone Tibetan for instance has t6ũ: ${ }^{\mathrm{L}}$ wa: ${ }^{\mathrm{H}}$, [42]).

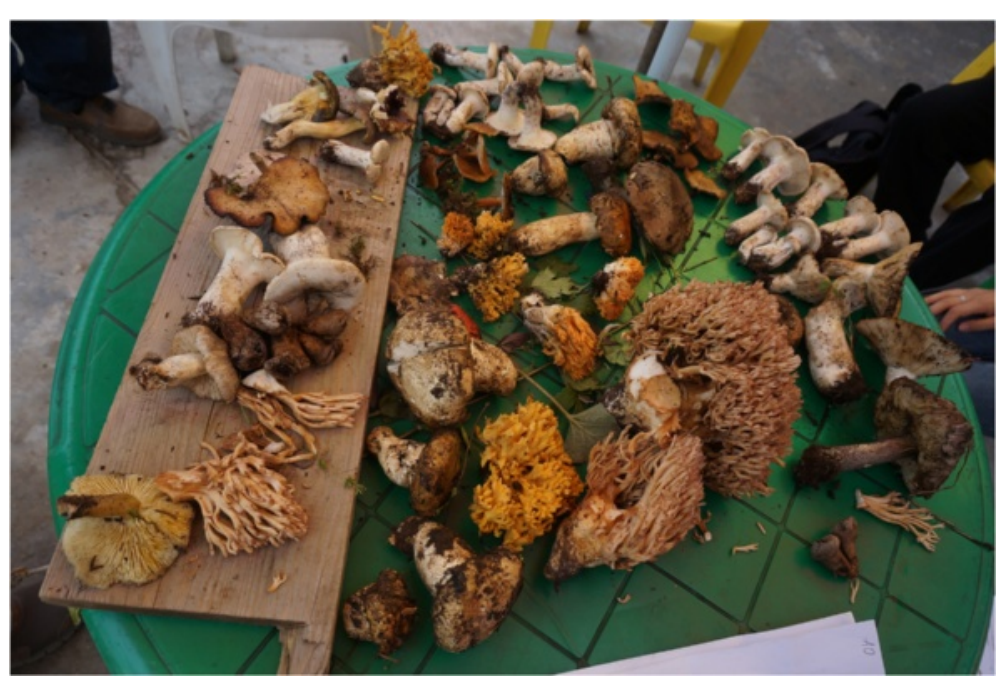

Fig. 8 A collection of edible and non-edible mushrooms ready for sorting 


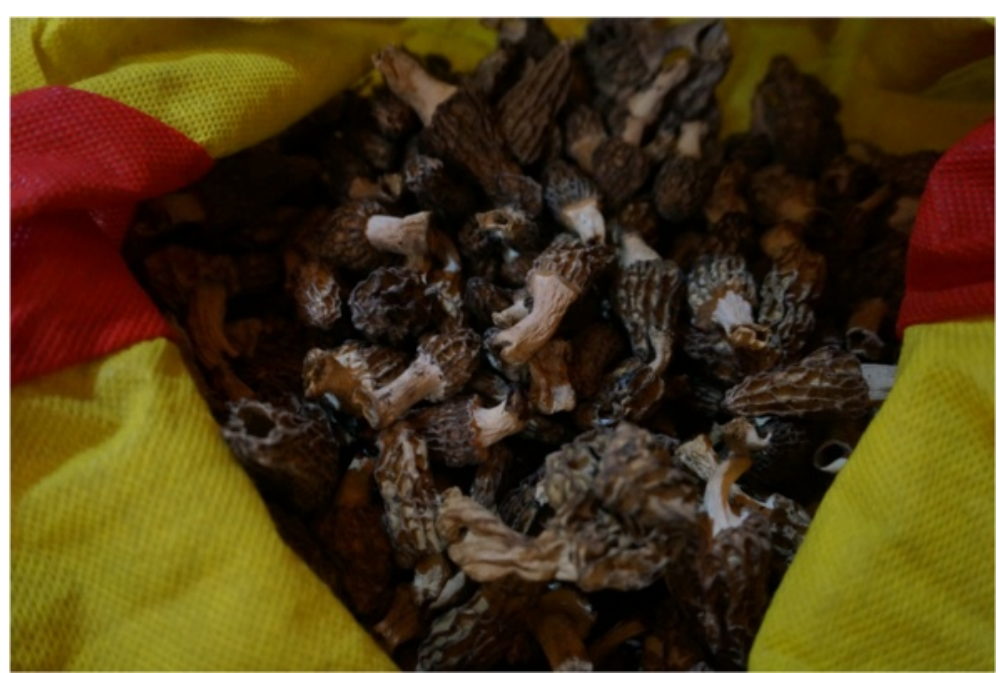

Fig. 9 Dried Morchella elata for winter use or for sale

The number of wild taxa eaten in the studied valley is relatively low, much lower than in the lower elevations and in the Qinling Mountains. However the main reason for this is the lower number of species available in a landscape dominated by nearly pure spruce forests. The average length of freelisted wild foods is quite long and similar to other places in the mountains of westerncentral China. On the other hand, a particularly large number of fungi are eaten, and in this case, the spruce forests are a favourable habitat for many mycorrhizal edible taxa.

Interestingly, the composition of the list of edible taxa highly resembles many areas of Eastern Europe [43-50]. The following similarities may be noted:

- the large number of fungi taxa consumed,
- similar genera or even species eaten (e.g., Rumex acetosa, Urtica dioica, Chenopodium album, Lactarius deliciosus, Agaricus campestris, Fragaria, Rubus, Sorbus, Ribes, Agaricus, Russula and Morchella),

- similar length of lists of fungi, fruits and wild vegetables consumed,

- a high appreciation of the sour taste, in contrast e.g., to Chinese people in the Qinling Mts. [7, 8].

However, the list of the collected taxa and their processing also displays features typical for other east Asian communities - e.g., the use of Pteridium aquilinum, drying wild vegetables for winter, a lack of preserves made of fruits and fungi. The list of species is quite similar to the edible plants used by Tibetan communities recorded by

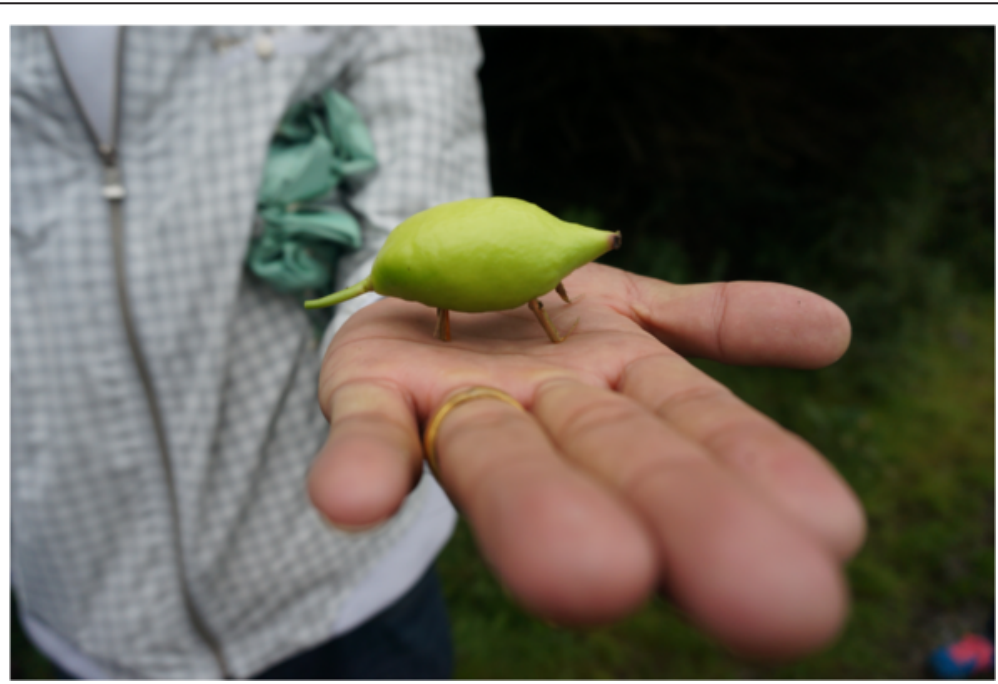

Fig. 10 Sinopodophyllum hexandrum is called 'toy pig' as apart from being eaten it is used for making miniature pigs, as children's toys 
Boesi in the vicinity of Litang (Sichuan) [1]. The people in Litang also eat Potentilla roots, Allium, Rosa shoots, Berberis flowers, Rheum stalks, Sinopodophyllum fruits (Fig. 10), Urtica, Chenopodium and Thlaspi shoots, food is spiced with Carum fruits etc. and Persicaria vivipara is used there under a nearly identical name $(r a m b u)$. A very similar composition of wild foods was also recorded in south-central Tibet in another study [4]. There, Rheum spp. stalks, P. anserina bulbs, Chenopodium album, Allium and Urtica are also eaten.

It should be emphasized that although edible mushrooms are highly appreciated in China, it is only in Yunnan that larger numbers of mushroom species are used for food (e.g., $[51,52])$. In the areas east of our study area, at lower elevations, in Gansu and Shaanxi, we recorded much lower numbers of species used and the very rare occurrence of drying mushrooms for winter use. For the moment, the use of over 20 fungi taxa in a wild valley makes the studied communities the most mycophilous places outside Yunnan. However this may simply result from the low number of ethnomycological studies in central Asia. For example it is worth noting that the number of edible fungi taxa recorded in this study (22) is similar to that used by Sherpa people in Nepal, on the other edge of the Tibetan Plateau (26 species) [53].

\section{Conclusions}

The studied community uses a nearly equal number of fungi, fruit and wild vegetable species. The list of taxa used is not very long for a rural mountain community in Asia, but this stems mainly from the low species richness of the vegetation surrounding the villages.

The ZhaganaTibetans are probably the most mycophilous ethnic group in China outside Yunnan.

The composition of wild food plant taxa is typical for Tibetan speaking areas (e.g., the use of rhubarb shoots, Potentilla anserina, Persicaria vivipara).

\section{Acknowledgments}

Many thanks to the inhabitants of the studied villages for their willingness in sharing information on the use of the species. The program was financially supported by the Forestry Research Foundation for the Public Service Industry of China $(2009,04004)$ and by the University of Rzeszów (Institute of Biotechnology and Basic Sciences, as well as a special grant from the rector of the university).

\section{Authors' contributions}

Field study and data processing: JK, YK, XJ, QG, NŁ, DL, ŁŁ. Fungi identification MP, Phonetic transcription - GJ. First draft of the paper - ŁŁ. All authors read and approved the final manuscript.

\section{Competing interests}

The authors declare that they have no competing interests.

\section{Author details}

'College of Forestry, Northwest A\&F University, Yangling, Shaanxi 712100, People's Republic of China. ${ }^{2}$ Yangling Vocational \& Technical College, Yangling, Shaanxi 712100, People's Republic of China. ${ }^{3}$ Forestry Academy of Bailongjiang Forestry Administration Bureau, Liangshui, Gansu 746010,
People's Republic of China. ${ }^{4}$ Department of Plant Taxonomy and Nature Conservation, University of Gdansk, Wita Stwosza 59, Gdańsk 80-308, Poland. ${ }^{5}$ Institute of Dendrology Polish Academy of Sciences, ul Parkowa 5, Kórnik 62-035, Poland. ${ }^{6}$ French National Centre for Scientific Research, Centre de recherches linguistiques sur l'Asie orientale, 2 rue de Lille, Paris 75007, France. 7 University of Glasgow (Bachelor of Art degree course), Glasgow, UK.

${ }^{8}$ Department of Botany, Institute of Applied Biotechnology and Basic Sciences, University of Rzeszów, Werynia 502, Kolbuszowa 36-100, Poland.

Received: 8 April 2016 Accepted: 17 May 2016

Published online: 01 June 2016

\section{References}

1. Boesi A. Traditional knowledge of wild food plants in a few Tibetan communities. J Ethnobiol Ethnomed. 2014;10(1):1.

2. Ju Y, Zhuo J, Liu B, Long C. Eating from the wild: diversity of wild edible plants used by Tibetans in Shangri-la region, Yunnan, China. J Ethnobiol Ethnomed. 2013;9(1):28.

3. Kang Y, Łuczaj L, Kang J, Wang F, Hou J, Guo Q. Wild food plants used by the Tibetans of Gongba Valley (Zhouqu county, Gansu, China). J Ethnobiol Ethnomed. 2014;10:20.

4. Malaisse F, Claus W, Drolkar P, Lopsang R, Wangdu L, Mathieu F. Ü Ethnomycology and Ethnobotany (South Central Tibet). Diversity, with emphasis on two underrated targets: plants used for dyeing and incense. Geo Eco Trop. 2012;36:185-99.

5. Boesi A. Plant categories and types in Tibetan materia medica. Tibet J. 2005: 30(4/1):65.

6. Boesi A. Plant knowledge among Tibetan populations. Memorie della Società Italiana di Scienze Naturali e del Museo Civico di Storia Naturale di Milano. 2005;33(1):33-48.

7. Liu Y, Dao Z, Yang C, Liu Y, Long C. Medicinal plants used by Tibetans in Shangri-la, Yunnan, China. J Ethnobiol Ethnomed. 2009;5:15.

8. Duncan MH. Customs and superstition of Tibetans. London: The Mitre Press; 1964

9. Rockhill WW. Diary of a journey through Mongolia and Tibet in 1891 and 1892. Washington: Smithsonian Institute; 1894.

10. Kang Y, Łuczaj Ł, Yes S, Zhang S, Kang J. Wild food plants and wild edible fungi of Heihe valley (Qinling Mountains, Shaanxi, central China): herbophilia and indifference to fruits and mushrooms. Acta Soc Bot Pol. 2012;81(4):405-13

11. Kang Y, Łuczaj $Ł$, Kang J, Zhang S. Wild food plants and wild edible fungi in two valleys of the Qinling Mountains (Shaanxi, central China). J Ethnobiol Ethnomed. 2013;9(1):26.

12. Maurizio A. Die Geschichte unserer Pflanzennahrung: von den Urzeiten bis zur Gegenwart. Berlin: Parey; 1927.

13. Johns T. With bitter herbs they shall eat it: chemical ecology and the origins of human diet and medicine. Tucson: University of Arizona Press; 1990.

14. Bharucha Z, Pretty J. The roles and values of wild foods in agricultural systems. Phil Trans R Soc B. 2010;365:2913-26.

15. Delang C. Not just minor forest products: the economic rationale for the consumption of wild food plants by subsistence farmers. Ecol Econ. 2006;59:64-73.

16. Turner NJ, Łuczaj $Ł J$, Migliorini P, Pieroni A, Dreon AL, Sacchetti LE, Paoletti MG. Edible and tended wild plants, traditional ecological knowledge and agroecology. Cr Rev Plant Sci. 2011;30(1-2):198-225.

17. Zou X, Huang F, Hao L, Zhao J, Mao H, Zhang J, Ren S. The socio-economic importance of wild vegetable resources and their conservation: a case study from China. Kew Bull. 2010;65:577-82.

18. Hu SY. Food plants of China. Hongkong: The Chinese University Press; 2005.

19. Ghorbani A, Langenberger G, Sauerborn J. A comparison of the wild food plant use knowledge of ethnic minorities in Naban River Watershed National Nature Reserve, Yunnan, SW China. J Ethnobiol Ethnomed. 2012;8:17.

20. Pemberton RW, Lee NS. Wild food plants in South Korea; market presence, new crops, and exports to the United States. Econ Bot. 1996;50(1):57-70.

21. Kosaka Y, Xayvongsa L, Vilayphone A, Chanthavong H, Takeda S, Kato M. Wild edible herbs in paddy fields and their sale in a mixture in Houaphan Province, the Lao People's Democratic Republic. Econ Bot. 2013;67(4):335-49.

22. Hosking R. A dictionary of Japanese food: ingredients \& culture. North Clarendon (Vermont, USA): Tuttle Publishing; 2014. 
23. Ogle BM, Hung PH, Tuyet HT. Significance of wild vegetables in micronutrient intakes of women in Vietnam: an analysis of food variety. Asia Pac J Clin Nutr. 2001;10(1):21-30.

24. Cruz-Garcia GS, Price LL. Weeds as important vegetables for farmers. Acta Soc Bot Pol. 2012;81(4):397-403.

25. Lin Y. Thebo. In: Sun JT-S, editor. Phonological profiles of little-studied Tibetic varieties. Taipei: Academia Sinica; 2014. p. 215-64.

26. Ning $X$, editor. Diebu County Annals. Lanzhou: Lanzhou University Press; 2010. p. 136-157, 820-828. [宁学义主编. 迭部县志兰州大学出版社: 兰州; 2010. pp. 136-157, 820-828].

27. Chock AK. J. F. Rock 1884-1962. Taxon. 1963;12(3):89-102.

28. Rock JF. Life among the Lamas of Choni: describing the mystery plays and butter festival in the monastery of an almost unknown Tibetan principality in Kansu Province, China. Nat Geog Mag. 1928;54:569-619.

29. Rock JF. Land of the Tebbus. Geog Jour. 1933;81:108-27.

30. Croll EJ. Research methodologies appropriate to rapid appraisal: a Chinese experience. IDS Bull. 1984;15(1):51-6.

31. Long $\mathrm{CL}$, Wang JR. Participatory rural appraisal: an introduction to principle, methodology and application. Kunming: Yunnan Science and Technology Press; 1996.

32. American Anthropological Association Code of Ethics. http://www.aaanet.org/ issues/policy-advocacy/upload/AAA-Ethics-Code-2009.pdf. Accessed 10 Mar 2016.

33. International Society of Ethnobiology Code of Ethics (with 2008 additions). http://ethnobiology.net/code-of-ethics/. Accessed 10 Mar 2016.

34. Sutrop U. List task and a cognitive salience index.Field. Methods. 2001; 3(13):263-76

35. Quinlan M. Considerations for collecting freelists in the field: examples from ethnobotany. Field Methods. 2005;17(3):219-34.

36. Łuczaj Ł, Kang Y, Kang J, Guillaume J. Nagrania nazw roślin w języku tebotybetańskim. Repozytorium Uniwersytetu Rzeszowskiego. http:// repozytorium.ur.edu.p//handle/item/1436.

37. The Plant List: a working list of all plant species. http://www.theplantlist. org/. Accessed 10 Mar 2016.

38. Index Fungorum. http://www.indexfungorum.org/. Accessed 10 Mar 2016.

39. Schocha CL, Seifertb K, Huhndorfc S, et al. Nuclear ribosomal internal transcribed spacer (ITS) region as a universal DNA barcode marker for Fungi. NPAS. 2012;109(16):6241-6.

40. Begerow D, Nilsson H, Unterseher M, Maier W. Current state and perspectives of fungal DNA barcoding and rapid identification procedures. Appl Microbiol Biotechnol. 2010;87:99-108.

41. GenBank. http://www.ncbi.nlm.nih.gov/. Accessed 1 Mar 2016

42. Jacques G. Cone. In: Sun JT-S, editor. Phonological profiles of little-studied Tibetic varieties. Taipei: Academia Sinica; 2014. p. 265-371.

43. Šimková K, Polesný Z. Ethnobotanical review of wild edible plants used in the Czech Republic. J Appl Bot Food Qual. 2015;88:49-67.

44. Schunko C, Vogl CR. Organic farmers use of wild food plants and fungi in a hilly area in Styria (Austria). J Ethnobiol Ethnomed. 2010;6:17.

45. Stryamets N, Elbakidze M, Ceuterick M, Angelstam P, Axelsson R. From economic survival to recreation: contemporary uses of wild food and medicine in rural Sweden, Ukraine and NW Russia. J Ethnobiol Ethnomed. 2015;11:53.

46. Łuczaj Ł, Stawarczyk K, Kosiek T, Pietras M, Kujawa A. Wild food plants and fungi used by Ukrainians in the western part of the Maramureş region in Romania. Acta Soc Bot Pol. 2015;84(3):339-46.

47. Kalle R, Söukand R. Historical ethnobotanical review of wild edible plants of Estonia (1770s-1960s). Acta Soc Bot Pol. 2012;81(4):271-8.

48. Dénes A, Papp N, Babai D, Czúcz B, Molnár Z. Wild plants used for food by Hungarian ethnic groups living in the Carpathian Basin. Acta Soc Bot Pol. 2012;81(4):381-96.

49. Łuczaj Ł, Szymański WM. Wild vascular plants gathered for consumption in the Polish countryside: a review. J Ethnobiol Ethnomed. 2007;3(1):1.

50. Łuczaj $Ł$, Nieroda Z. Collecting and learning to identify edible fungi in southeastern Poland: age and gender differences. Ecol Food Nutr. 2011; 50(4):319-36.

51. Boa ER. Wild edible fungi: a global overview of their use and importance to people. Rome: Food \& Agriculture Org.; 2004.

52. Wang $X$, Liu P. Resources investigation and studies on the wild commercial fungi in Yunnan. Chin Biodivers. 2001;10(3):318-25.

53. Giri A, Rana R. Ethnomycological knowledge and nutritional analysis of some wild edible mushrooms of Sagarmatha national Park (SNP), Nepal. J Nat Hist Mus. 2009;23:65-77.

\section{Submit your next manuscript to BioMed Central and we will help you at every step:}

- We accept pre-submission inquiries

- Our selector tool helps you to find the most relevant journal

- We provide round the clock customer support

- Convenient online submission

- Thorough peer review

- Inclusion in PubMed and all major indexing services

- Maximum visibility for your research

Submit your manuscript at www.biomedcentral.com/submit 